\title{
Prognostic Role of Pretreatment Plasma D-Dimer in Patients with Solid Tumors: a Systematic Review and Meta-Analysis
}

\author{
Wenhan Lia Yao Tang ${ }^{b}$ Yongchun Song ${ }^{a}$ Szu Hao Chen ${ }^{c}$ Navard Sisliyan ${ }^{d}$ \\ Ming $\mathrm{Ni}^{\mathrm{e}} \mathrm{Hao}_{\text {Zhang }}{ }^{\mathrm{a}}$ Qingnuo Zeng ${ }^{\mathrm{a}} \mathrm{Bin} \mathrm{Hou}^{\mathrm{a}} \mathrm{Xin} \mathrm{Xie}^{\mathrm{a}}$ Dongmin Chang ${ }^{\mathrm{a}}$ \\ aDepartment of Surgical Oncology, the First Affiliated Hospital of Xi'an Jiaotong University, Xi'an, \\ Shaanxi, 'Department of general surgery, Xi'an No.3 hospital, Xi'an, Shaanxi, China; 'Department of \\ Molecular and Medical Pharmacology, Crump Institute for Molecular Imaging, California NanoSystems \\ Institute, University of California, Los Angeles, CA, 'Departments of Medicine, University of California, \\ Los Angeles, CA, USA; 'Department of liver surgery, the first affiliated hospital of Nanjing Medical \\ University, Nanjing, Jiangsu, China
}

\section{Key Words}

D-dimer • Solid tumors • Prognosis • Meta-analysis

\begin{abstract}
Background/Aims: Elevated pretreatment plasma D-dimer level has been reported as an unfavorable prognostic indicator in several malignancies. The aim of this meta-analysis was to evaluate the prognostic value of elevated D-dimer level in solid tumors. Methods: A comprehensive search of electronic databases up to June 10, 2017 was carried out by two independent reviewers. We included studies exploring the association between pretreatment plasma D-dimer level and patients' survival outcomes in solid tumors. Overall survival (OS) was regarded as primary outcome and progression-free survival (PFS), disease-free survival (DFS) as well as cancer-specific survival (CSS) were chosen as secondary outcomes. Hazard ratio and $95 \%$ confidence interval (CI) were extracted directly or indirectly from included studies. Results: 49 studies with 13001 patients were included in our meta-analysis. Elevated D-dimer was markedly associated with poor OS (pooled HR $=1.90,95 \% \mathrm{CI}=1.63-2.20, \mathrm{P}<$ 0.001). The effect was observed in all different tumor sites, disease stages, cut-off values and ethnicities. Meanwhile, patients with a high plasma D-dimer had a shorter PFS (HR $=1.46,95 \%$ $\mathrm{CI}=1.22-1.76 ; \mathrm{P}<0.001)$, DFS $(\mathrm{HR}=2.02,95 \% \mathrm{CI}=1.56-2.62)$ and $\mathrm{CSS}(\mathrm{HR}=2.04,95 \% \mathrm{CI}=$ 1.58 - 2.64). Conclusions: Analysis of the pretreatment plasma D-dimer might provide useful information to predict prognosis in patients with solid tumors.




\section{Cellular Physiology Cell Physiol Biochem 2018;45:1663-1676 \begin{tabular}{ll|l} 
and Biochemistry Published online: February 27, 2018 & $\begin{array}{l}\text { (c) } 2018 \text { The Author(s). Published by S. Karger AG, Basel } \\
\text { www.karger.com/cpb }\end{array}$ \\
\hline
\end{tabular}}

Li et al.: Prognositc Role of D-Dimer in Solid Tumors: a Meta-Analysis

\section{Introduction}

The hemostatic system plays an important role in coordinating the balance between bleeding and clot formation. Interestingly, abnormal activation of coagulation and fibrinolysis is frequently present in cancer patients, especially in patients with metastatic disease [19]. The spectrum of hemostatic abnormalities ranges from massive thromboembolism to abnormal coagulation parameters in the absence of clinical symptoms [10]. This phenomenon may due to the fact that tumor cells could possess strong procoagulant activities, resulting in coagulation system's local activation and fibrin deposition [11]. The formation of plateletfibrin-tumor cell creates an extracellular microenvironment that can facilitate tumor cell growth and survival.

D-dimer, a plasmin-mediate degradation product of cross-linked fibrin, is a biomarker for routine use in clinical practice of deep venous thrombosis (DVT), disseminated intravascular coagulation (DIC) and pulmonary embolism (PE). Circulating D-dimer may also elevated in patients with coronary artery disease, trauma, pregnancy and inflammatory diseases [12, 13]. Recent articles reported that plasma D-dimer was markedly elevated in patients with different malignancies before treatment, including lung cancer [1-3], prostate cancer [4, 5], cervical cancer [6, 7], breast cancer [8] and colorectal cancer [9]. However, the prognosis role of D-dimer in cancer is still controversial. Some reported that an elevated D-dimer level before treatment is associated with a more advanced tumor stage or a more advanced clinical progression therefore it may indicate a worse prognosis. While others claimed the connection was insignificant. For now, there has been no systematic attempt to explore the consistency and magnitude of its prognostic value. Thus, we conducted this systemic review and meta-analysis to elucidate the prognostic role of D-dimer in solid tumors.

\section{Materials and Methods}

\section{Data sources}

The present meta-analysis was strictly performed in line with the Preferred Reporting Items for Systematic Reviews and Meta-Analyses (PRISMA) statement [14]. We conducted a comprehensive review of Pubmed database up to June 10 by two independent reviewers. The key terms of this analysis include "cancer", "D-dimer" and "prognosis". Meanwhile, we searched abstracts of the American Society of Clinical Oncology (ASCO) using the same search terms. Additionally, an independent search of Embase, the web of science and Cochrane Library database was also carried out in case of missing any other information. The search results were limited to human studies published in English language.

\section{Study selection}

Studies eligible for this analysis had to meet the following criteria: (1) prospective or retrospective cohort studies of patient with non-hematologic solid tumors; (2) assessment of the prognostic effect of pretreatment D-dimer on overall survival (OS), progression-free survival (PFS), disease-free survival (DFS) or cancer-specific survival (CSS). (3) containing sufficient data to extract or calculate the hazard ratio (HR) and its 95\% confidence interval (CI). Articles were excluded if they contained any of the following criteria: (1) articles of patient with hematologic malignancies; (2) duplicate publication; (3) animal experiments, non-English papers; (4) comments, reviews, case reports or meta-analyses containing no original data; (4) insufficient data for extracting or calculating of HR and its 95\% CI.

Two independent reviewers (YC Song, H Zhang) evaluated all the selected titles and abstracts to exclude duplicated publications and apparent irrelevant studies. Then the results were pooled and full texts were retrieved to assess eligibility. Any discrepancies were resolved by consensus. Corresponding authors were contacted to clarify missing or ambiguous data.

Data extraction

Two investigators (WH Li, X Xie) independently extracted data from all included studies by using predesigned data-abstraction form. The following data were collected: title of publication, first author's 
name, research institution, year of publication, country, study design (retrospective, prospective), characteristics of study objects (sample size, age, sex), primary tumor site, disease stage (nonmetastatic, metastatic, mixed), tumor stage (I, II , III, IV), follow-up period, cut-off value, study endpoints (OS, DFS/ RFS and CSS assessed as HRs with corresponding 95\% CIs and/or P values), survival analysis models (multivariate, univariate).

\section{Quality assessment}

Two independent investigators (QN Zeng, B Hou) assessed the quality of the included studies according to Study OBservational studies in Epidemiology (STROBE) recommendations with an adjustment to the specific characteristics of the study [15]. The score of each article was assessed based on whether the following criteria were clearly stated: study hypothesis, tumor stage, description of eligibility criteria, whether patients with VTE or receive anticoagulation therapy were excluded, outcome endpoints definition, confounding factors considered in multivariate analysis, follow-up period, limitations and bias. We ranked the included studies according to the criteria considered in the analysis (quality score: 0 - 8). All discrepancies were discussed and resolved with consensus.

\section{Statistical analysis}

All statistical analysis was performed with the STATA software package (version 13.1) (Stata Corp LP, College Station, TX). We considered OS was the primary endpoint in this analysis and PFS, DFS and CSS were secondary outcomes. HRs with corresponding 95\% CIs were directly extracted from each study if it is feasible. For studies only providing Kaplan-Meier survival curves, data were calculated by using software Engauge Digitizer Version $4.1[16,17]$. HR values $>1$ indicated a poor survival with elevated serum D-dimer while HR values $<1$ implied a survival benefit with increased serum D-dimer, the result was considered statistically significant if the $95 \%$ CI did not overlap 1 . The heterogeneity of data among included studies was examined using Cochran's Q-test and Higgins $-\mathrm{I}^{2}$ statistic. We define heterogeneity was insignificant if $\mathrm{I}^{2}$ value $<50 \%$ and we use a fixed-effect model to calculate the parameters. If $\mathrm{I}^{2}$ value $\geq 50 \%$, we consider there exists a significant heterogeneity and a random-effect model will be applied to the data. Subgroup analyzes were conducted according to (1) primary tumor site, (2) disease stage (non-metastatic, metastatic, mixed) (3) D-dimer cut-off value, (4) patients' ethnicity (Asian, Non-Asian), (5) study design (retrospective, prospective). Moreover, sensitivity analyzes were performed to explore the impacts on pooled effect by removing the study sequentially. Meanwhile, metaregression was performed to assess whether moderator variables could influence the pool effect size for OS. Assessment of publication bias was shown by funnel plots, confirmed by Egger's tests and Begg's test. If publication bias is significant, the Duval and Tweedie's "Trim and Fill" method will be used to estimate a corrected effect size after adjustment [18]. Two-sided $\mathrm{P}$ values $<0.05$ were considered statistically significant.

\section{Results}

Study characteristics

3175 publications were initially identified following

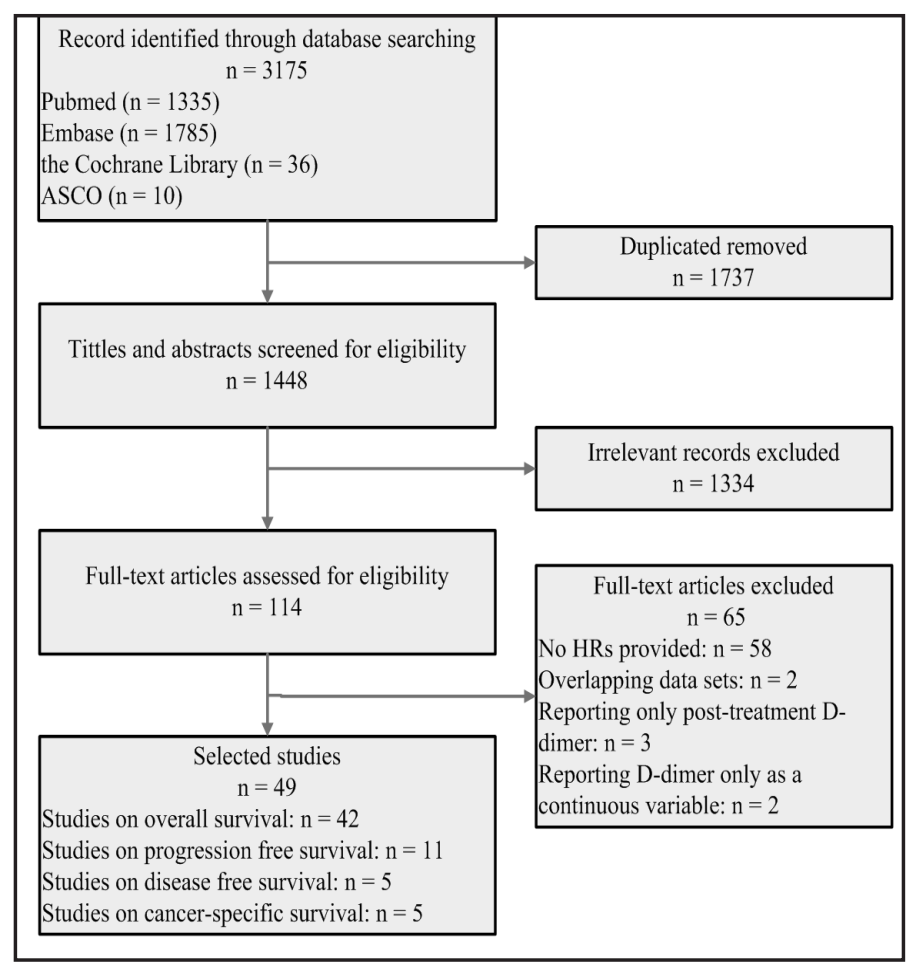

Fig. 1. Flowchart of the study selection process. 
our search strategy. After removing duplicated publications, 1448 studies were selected for further analysis. We next screened the titles and abstracts of each article. Reviews, case reports, in vitro studies and non-human studies were excluded in our analysis. Consequently, 114 eligible studies received full text evaluation. Among them, 65 studies with insufficient survival data were excluded from this analysis. Thus, a total of 49 studies with 12649 patients fulfilled our inclusion criteria and were enrolled in our study. The selection process is shown in Fig. 1.

Table 1 summarized the baseline characteristics of the included studies. Briefly, the publication time of included studies was between 1997 and 2017. Thirty studies collected data retrospectively while the rest used a prospective study design. D-dimer concentration was measure by either immunoturbidimetric assay (ITM) or Latex Test with various cut-off values among those studies. Of all eligible studies, Fifteen studies reported on lung cancer [2, $3,19-31]$, followed by colorectal cancer $(n=9)[9,21,32-38]$, gastric cancer $(n=5)[21,39$ 42], pancreatic cancer $(n=5)$ [21, 43-46], ovarian cancer $(n=3)$ [47-49], esophageal cancer $(\mathrm{n}=3)$ [50-52], cervical cancer $(\mathrm{n}=2)[6,7]$, renal cancer $(\mathrm{n}=2)[21,53]$, sarcoma cancer $(\mathrm{n}$ $=2)[54,55]$, brain cancer $(n=2)[21,56]$, endometrial cancer $(n=2)[57,58]$, breast cancer $(\mathrm{n}=1)[21]$, nasopharyngeal cancer $(\mathrm{n}=1)$ [59], prostate cancer $(\mathrm{n}=1)[21]$, malignant

Table 1. Main features of the included studies in the meta-analysis. CRC $=$ colorectal cancer; HCC = hepatocellular carcinoma; GBM = glioblastoma multiforme; ITM = immunoturbidimetry; NA = not available; NPC = nasopharyngeal carcinoma; NSCLC = non-small cell lung cancer; SCLC $=$ small cell lung cancer

\begin{tabular}{|c|c|c|c|c|c|c|c|c|c|c|c|c|c|}
\hline Author & Country & $\begin{array}{l}\text { Study Design } \\
\end{array}$ & Disease Stte & Stage & $\begin{array}{l}\text { Clinical setting } \\
\end{array}$ & Case Na & $\begin{array}{l}\text { Afereyears) } \\
\text { Median/mean (range) }\end{array}$ & Follow-up Median months (range) & $\begin{array}{cc}\text { cutoff }(\mathrm{ng} / \mathrm{ml}) \\
\end{array}$ & Detection Method & Outtome & HRs provided from & 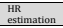 \\
\hline$L[57]$ & China & $\mathrm{R}$ & Endometrial & $t-N$ & Mixed & 282 & $53(21-67]$ & 51.2(0.3.-71.8) & 1500 & Latex Test & os & MV & $\begin{array}{c}\text { Survivas } \\
\text { curves } \\
\end{array}$ \\
\hline Nakamura [58] & Japan & $\mathrm{R}$ & Endometrial & $\mathrm{t}-\mathrm{N}$ & Mixed & 108 & $60(27-87)$ & NA & 1000 & Latex Test & os/PFs & MV/WV & $\begin{array}{l}\text { Hi R and } \\
95 \% \mathrm{CI}\end{array}$ \\
\hline Oya [38] & Japan & $\mathrm{R}$ & CRC & $\mathrm{t}-\mathrm{W}$ & Mixed & 93 & $62.7(27-84)$ & 54.7 & 500 & Latex Test & os & $M V / W$ & $\begin{array}{l}\text { HR and } \\
95 \% \% \mathrm{CI}\end{array}$ \\
\hline Blackwel| [32] & usA & ${ }^{\mathrm{P}}$ & CRC & iv & Metastatic & 104 & 61 (23-85) & NA & 130 & NA & os & Mv & 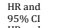 \\
\hline Yamamoto [34] & Japan & $\mathrm{R}$ & $\mathrm{CRC}$ & w & Metasatitic & 42 & NA & 14 & 1000 & ELLSA & css & MV/W & $\begin{array}{l}\text { HA and } \\
95 \% \text { CI }\end{array}$ \\
\hline Stender [9] & Denmark & $\mathrm{p}$ & $\mathrm{CRC}$ & $\mathrm{t}-\mathrm{W}$ & Mixed & 157 & $68(33.94)$ & NA & 300 & Latex test & os & MV & 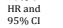 \\
\hline Tellioglu [33] & usA & ${ }^{\mathrm{p}}$ & CRC & w & Metasatic & 242 & 63 & ${ }^{22}$ & 1000 & NA & os & Mv & 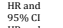 \\
\hline Zhu [35] & china & $\mathrm{R}$ & $\mathrm{CRC}$ & w & Metasatatic & 74 & $55.5(31-74)$ & $18.4(6.3 .3 .30 .4)$ & 1900 & Laterextest & os/PFs & MV/W & 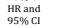 \\
\hline Tekesin [36] & Turkey & $\mathrm{P}$ & $\mathrm{CRC}$ & H-W & Mixed & 134 & $62.5(31-84)$ & $18(4-31)$ & 960 & ITM & os & $\mathrm{MV} / \mathrm{WV}$ & $\begin{array}{l}\text { HR and } \\
\text { 955\% CI }\end{array}$ \\
\hline Hong [37] & china & $\mathrm{p}$ & $\mathrm{CRC}$ & I-WII & Non-metasatatic & 505 & 63 (27-93) & $43(4-62)$ & 216 & $\mathrm{rm}$ & os & MV/WV & $\begin{array}{l}\text { HF and } \\
\text { 95 anc }\end{array}$ \\
\hline Liu [43] & china & $\mathrm{R}$ & Pancreatic & 1-III & Non-metastatic & 168 & $60(34-83)$ & $14(3-48)$ & 500 & тाM & os & MV & $\begin{array}{l}\text { HA and } \\
95 \% \text { CI }\end{array}$ \\
\hline $\operatorname{sun}[44]$ & China & R & Pancreatic & $1-\mathrm{N}$ & Mixed & 139 & $58.9(33-80)$ & NA & 600 & NA & os & MV & $\begin{array}{l}\text { HiR Rand } \\
95 \% \mathrm{CI}\end{array}$ \\
\hline Stender [45] & Denmark & $\mathrm{p}$ & Pancreatic & $1-\mathrm{N}$ & Mixed & 95 & NA & NA & 1000 & Latex Test & os & MV/LV & $\begin{array}{l}\text { HA Rand } \\
\text { 95 as\% CI }\end{array}$ \\
\hline $\mathrm{CaO}[46]$ & china & $\mathrm{R}$ & Pancreatic & I-II & Non-metasatatic & 119 & NA & $\mathrm{NA}(3-66)$ & 500 & ттм & os/PFS & uv & $\begin{array}{l}\text { Survival } \\
\text { curves }\end{array}$ \\
\hline Zhu [26] & china & P & scLc & $\mathrm{NA}$ & Mixed & 74 & 57 (42-800) & $11.5(3.5 .61)$ & 550 & ITM & ${ }_{\mathrm{os} / \mathrm{PFS}}$ & MV & $\begin{array}{l}\text { Hit and } \\
95 \% \text { CI }\end{array}$ \\
\hline Chen [25] & China & R & scLC. & $1-N$ & Mixed & 393 & $57(51-64)$ & $12(3-108)$ & 500 & ттм & os/PFS & MV/WV & $\begin{array}{l}\text { Hit and } \\
95 \% \text { CI }\end{array}$ \\
\hline Masago [20] & Japan & p & NSCLLC & IIBB-IV & Mixed & 99 & 67 (35-88) & NA & 600 & ELLSA & os & MV & $\begin{array}{l}\text { Hit and } \\
95 \% \text { CI }\end{array}$ \\
\hline Zhang [22] & china & $\mathrm{R}$ & NSCLLC & I-IIIA & Non-metasatatic & 232 & $61(30-86)$ & $47(0-64)$ & 300 & ттм & os & mV & $\begin{array}{l}\text { HA and } \\
\text { 95\% CI }\end{array}$ \\
\hline Fukumoto [3] & Japan & $\mathrm{R}$ & NSCLCC & I-III & Non-metasatatic & 237 & $69(31-85)$ & $51.6(1-76)$ & 500 & NA & os & MV/WN & $\begin{array}{l}\text { HA and } \\
\text { 95 anc CI }\end{array}$ \\
\hline Jiang [23] & China & $\mathrm{R}$ & NSCLC & I-III & Non-metastatic & 184 & $60(40-78)$ & $18.5(9.5 .32)$ & 550 & $\mathrm{rTM}$ & DFS & MV & 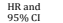 \\
\hline Wang [2] & China & $\mathrm{p}$ & NSSLLC & w & Metasatatic & 1931 & NA & $18(2-48)$ & 500 & $\mathrm{rrm}$ & os & Mv & $\begin{array}{l}\text { HA Rand } \\
\text { 95 as\% CI }\end{array}$ \\
\hline Ge [24] & china & p & NSCLLC & IIBB-VV & Mixed & 82 & $64(44-72)$ & $\mathrm{NA}$ & 550 & ITM & PFS & MV & $\begin{array}{l}\text { Hit and } \\
\text { 95:5 CI }\end{array}$ \\
\hline Han [28] & china & $\mathrm{R}$ & NSCLCC & $1-\mathrm{N}$ & Mixed & 173 & 57 & 8 & 500 & NA & os & Mv & $\begin{array}{l}\text { HA and } \\
\text { 95 anc CI }\end{array}$ \\
\hline Guo [27] & china & p & NSCLLC & IIBB-IV & Mixed & 119 & NA & NA & 996 & $\mathrm{rrm}$ & os & $\mathrm{MV} / \mathrm{WN}$ & $\begin{array}{l}\text { Hit and } \\
\text { } 95 \% \text { CI }\end{array}$ \\
\hline Diao [39] & China & $\mathrm{R}$ & Gastric & t-W & Mixed & 1042 & NA & NA & 1500 & $\mathrm{rm}$ & os/DFs & мv & $\begin{array}{l}\text { HF and } \\
\text { 9r5\% a CI }\end{array}$ \\
\hline Liu [42] & China & $\mathrm{P}$ & Gasstric & t-N & Mixed & 247 & NA & NA & 1465 & NA & os & MV/WV & $\begin{array}{l}\text { HR and } \\
\text { 9r5\% CI }\end{array}$ \\
\hline $\mathrm{Go}_{0}[40]$ & Korea & $\mathrm{R}$ & Gastric & v & Metasatatic & 46 & NA & $16.2(2.2-2.5 .8)$ & 1500 & тाм & os & MV & 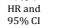 \\
\hline Kanda [41] & Japan & $\mathrm{R}$ & Gastric & II-III & Non-metastatic & 126 & NA (26-96) & NA & 1000 & Latex Test & os & uv & $\begin{array}{l}\text { Survuval } \\
\text { curves }\end{array}$ \\
\hline Diao [50] & china & $\mathrm{P}$ & Esophageal & $\mathrm{t}-\mathrm{N}$ & Mixed & 66 & NA & $\mathrm{NA}$ & 1500 & $\mathrm{rTM}$ & os & MV & $\begin{array}{l}\text { He and } \\
\text { He and } \\
95 \% \text { CI }\end{array}$ \\
\hline Zhang [52] & china & $\mathrm{R}$ & Esophageal & I-WII & Non-metasatatic & 468 & $60(36-81)$ & $49.1(3.2-114.5)$ & 207 & NA & oS/DFS & uv & $\begin{array}{l}\text { HA and } \\
95 \% \text { CI }\end{array}$ \\
\hline Feng [51] & china & $\mathrm{R}$ & Esophageal & I-III & Non-metasatatic & 337 & $59(36-80)$ & $\mathrm{NA}$ & 500 & ттм & css & MV/W & $\begin{array}{l}\text { HA and } \\
95 \% \text { CI }\end{array}$ \\
\hline Erdem [53] & Turkey & p & Renal & $1-N$ & Mixed & 128 & $58(21-83)$ & $36.5(1-88)$ & 760 & NA & oS/DFs/CSS & MV & $\begin{array}{l}\text { HA and } \\
95 \% \text { CI }\end{array}$ \\
\hline Sakurari [49] & Japan & $\mathrm{R}$ & ovarian & $\mathrm{H}-\mathrm{IV}$ & Mixed & 134 & $56.5(31-88)$ & $53(7-106)$ & 2000 & Latex Test & os & MV & $\begin{array}{l}\text { HF and } \\
95 \% \text { CI }\end{array}$ \\
\hline $\operatorname{Man}[48]$ & China & $\mathrm{R}$ & ovarian & $\mathrm{t}-\mathrm{N}$ & Mixed & 190 & 55 (225-83) & $48(2-150)$ & 300 & ттм & os/PFs & MV & $\begin{array}{l}\text { HA and } \\
\text { 9r5\% al }\end{array}$ \\
\hline Liu [47] & China & $\mathrm{R}$ & ovarian & $1-N$ & Mixed & 125 & $51(25 .-73)$ & $49(5-85)$ & 300 & ттм & os/PFs & MV/WN & $\begin{array}{l}\text { HA and } \\
95 \% \text { CI }\end{array}$ \\
\hline Moril [54] & Japan & ${ }_{\mathrm{R}}$ & Sarcoma & $\mathrm{NA}$ & NA & 85 & $55.7(9-95)$ & $23(6-50)$ & 800 & Latex Test & PFS & мv & 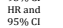 \\
\hline $\operatorname{Raj}[55]$ & usA & $\mathrm{R}$ & Sarcoma & $\mathrm{NA}$ & Mixed & 45 & $39(16-65)$ & $60(9-106)$ & 500 & Fluorescence immunoassay & os & MV & $\begin{array}{l}\text { HHand } \\
\text { } 95 \% \text { a CI }\end{array}$ \\
\hline Luo [7] & China & $\mathrm{R}$ & cervical & $\mathrm{t}-\mathrm{N}$ & Mixed & 296 & $45[25 .-79]$ & NA $(1-60)$ & 500 & $\mathrm{rrm}$ & os & MV & $\begin{array}{l}\text { HA Rand } \\
\text { 95 as\% CI }\end{array}$ \\
\hline Nakamura [6] & Japan & $\mathrm{R}$ & cervical & $1-\mathrm{N}$ & Mixed & 129 & $60(22-89)$ & NA & 1000 & Latex Test & os/PFS & $M V / W$ & $\begin{array}{l}\text { Hit and } \\
95 \% \text { CI }\end{array}$ \\
\hline Taguch [29]] & Japan & $\mathrm{p}$ & NSCLLC+SCLLC & $\mathrm{NA}$ & $\mathrm{NA}$ & 70 & $65[20-83)$ & $15(2-66)$ & 150 & ELISA & css & MV & $\begin{array}{l}\text { HA Rand } \\
\text { 95 as\% CI }\end{array}$ \\
\hline Ferrigno[30] & Italy & $\mathrm{p}$ & NSCLC+CSCLCC & $\mathrm{t}-\mathrm{N}$ & Mixed & 343 & $68[(39-86)$ & 8.5 & 500 & $\mathrm{rTM}$ & os & uv & $\begin{array}{l}\text { Survival } \\
\text { curves }\end{array}$ \\
\hline Buccher [31] & Italy & $\mathrm{R}$ & NSCLLC+SCLLC & 1.W & Mixed & 826 & $67(35-89)$ & 7.9 & 1000 & IтM & os & Mv & $\begin{array}{l}\text { Hit and } \\
95 \% \text { a CI }\end{array}$ \\
\hline \multirow[t]{3}{*}{ Altiay [19] } & Turkey & $\mathrm{P}$ & NSLLC+SCLCC & III-N & Mixed & 78 & 61 (37-82] & NA & 650 & ELISA & os & MV/UV & $\begin{array}{l}\text { HF and } \\
95 \% \mathrm{Cl}\end{array}$ \\
\hline & & & Breast & & & 157 & & & & Latex Test & & & \\
\hline & & & $\begin{array}{l}\text { Lung } \\
\text { Gastric }\end{array}$ & & & $\begin{array}{l}182 \\
50\end{array}$ & & & & & & & \\
\hline \multirow[t]{4}{*}{ Ay [21] } & Austria & ${ }^{\mathrm{P}}$ & $\begin{array}{l}\text { CRC } \\
\text { Pancreatic }\end{array}$ & NA & Mixed & $\begin{array}{l}133 \\
74\end{array}$ & NA & 24 & NA & & os & uv & $\begin{array}{l}\text { HR and } \\
95 \% \% \mathrm{CI}\end{array}$ \\
\hline & & & Renal & & & 37 & & & & & & & \\
\hline & & & Prostate & & & 133 & & & & & & & \\
\hline & & & Brain & & & 148 & & & & & & & \\
\hline Chen [59] & China & $\mathrm{R}$ & NPC & $\mathrm{t}-\mathrm{W}$ & Non-metastatic & 717 & $47(29.71)$ & $31(24-42)$ & 800 & $\mathrm{rTM}$ & $\mathrm{oS} / \mathrm{DFS}$ & MV/W & $\begin{array}{l}\text { HF and } \\
955 \% \mathrm{CI}\end{array}$ \\
\hline Watanabe [61] & Japan & $\mathrm{R}$ & Cholangibcarcinoma & $\mathrm{H}-\mathrm{V}$ & Non-metastatic & 55 & $\mathrm{NA}(36-84)$ & 48 & 1300 & NA & $\mathrm{PFS} / \mathrm{CSS}$ & uv & $\begin{array}{l}\text { Survival } \\
\text { curves }\end{array}$ \\
\hline Desch [60] & Germany & $\mathrm{R}$ & Malignant melanoma & $1-N$ & Mixed & $\begin{array}{l}81 \text { for of } \\
100 \text { or } \mathrm{PPS}\end{array}$ & $64[21 \cdot 94]$ & 60 & 600 & $\mathrm{rrm}$ & os/PFS & $\mathrm{MV} / \mathrm{WN}$ & $\begin{array}{l}\text { Hit and } \\
95 \% \text { CI }\end{array}$ \\
\hline Liw [62] & china & $\mathrm{R}$ & нсс & $1-\mathrm{N}$ & Non-metastatic & 192 & $46(\mathrm{NA})$ & 48 & 700 & NA & os & MV/W & $\begin{array}{l}\text { HRR and } \\
95 \% \text { CI }\end{array}$ \\
\hline Hoke [56] & Austria & $\mathrm{P}$ & Gвм & $\mathrm{NA}$ & Non-metastatic & 23 & $64.3(48.6 .6-6.62)$ & 7.3 & 1000 & Latee Test & os & MV/WV & 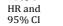 \\
\hline
\end{tabular}


melanoma $(\mathrm{n}=1)[60]$, cholangiocarcinoma $(\mathrm{n}=1)$ [61] and hepatobiliary cancer $(\mathrm{n}=1)$ [62]. Thirteen studies only chose non-metastatic cancer patients as research subjects while six studies only enrolled cancer metastatic patients. Twenty-seven studies reported on patients with both non-metastatic and metastatic disease. Forty-two studies used OS, nine studies used PFS, five studies used DFS and five studies used CSS as the end points, respectively. We measured the methodological quality of each included article and the results are shown in Table 2 . The included studies had a mean score of 6.47 (range 5 to 8).

\section{Primary outcome: overall} survival

Forty-two studies, including 11412 patients, provided appropriate data for OS analysis. Thirty-seven of those studies, comprising a total of 10176 patients, provided multivariable analysis HRs for OS and were adopted in our main quantitative analysis. As shown in Fig. 2, a high pretreatment D-dimer was significantly associated with shorter OS in a randomeffects model (pooled HR = $1.90,95 \% \mathrm{CI}=1.63-2.20, \mathrm{P}<0.001)$ with significant heterogeneity among studies $\left(\mathrm{I}^{2}=\right.$ $75.1 \%, \mathrm{P}<0.001)$.

Subgroup analyses were performed according to different tumor site. As shown in Fig. 3, We found that the highest predicting effect of elevated baseline D-dimer on OS was endometrial cancer (pooled $\mathrm{HR}=21.7,95 \% \mathrm{CI}=8.82-50.36, \mathrm{P}<0.001$ ), followed by cervical cancer (pooled HR $=2.23,95 \% \mathrm{CI}=1.30-3.82, \mathrm{P}=0.003$ ) and gastric cancer (pooled HR $=1.92,95 \% \mathrm{CI}=1.41-2.60, \mathrm{P}<0.001)$. High heterogeneity was observed among studies
Table 2. Methodological quality of 49 full-text studies included in the meta-analysis

\begin{tabular}{|c|c|c|c|c|c|c|c|c|}
\hline $\begin{array}{l}\text { Hypothesis and//or } \\
\text { objective(s) stated }\end{array}$ & $\begin{array}{l}\text { Tumorr } \\
\text { clearly } \\
\text { described }\end{array}$ & $\begin{array}{l}\begin{array}{l}\text { Clear description of } \\
\text { eligbility criteria }\end{array} \\
\end{array}$ & 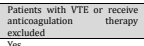 & 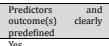 & 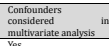 & $\begin{array}{l}\text { Follow-up period reported } \\
\text { and olon o enought for } \\
\text { outcomes to occur } \\
\end{array}$ & 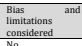 & $\begin{array}{l}\text { Quality } \\
\text { Scrore } \\
8 \text { 8 }\end{array}$ \\
\hline $\begin{array}{l}\text { Yess } \\
\text { Yes }\end{array}$ & $\begin{array}{l}\text { Yivs } \\
\text { Yes } \\
\text { Yes }\end{array}$ & $\begin{array}{l}\text { Yes } \\
\text { No }\end{array}$ & $\begin{array}{l}\text { Yes } \\
\text { No }\end{array}$ & $\begin{array}{l}\text { Yes } \\
\text { Yes }\end{array}$ & $\begin{array}{l}\text { Yes } \\
\text { Yes }\end{array}$ & $\begin{array}{l}\text { Yes } \\
\text { No }\end{array}$ & $\begin{array}{l}\text { No } \\
\text { Yes }\end{array}$ & $\begin{array}{l}7 \\
5\end{array}$ \\
\hline Yes & Yes & Yes & Yes & Yes & Yes & № & № & 6 \\
\hline Yes & Yes & № & Yes & Yes & Yes & Yes & Yes & ? \\
\hline $\begin{array}{l}\text { Yes } \\
\text { Yes } \\
\text { Yos }\end{array}$ & 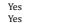 & $\begin{array}{l}\text { Yes } \\
\text { No }\end{array}$ & $\begin{array}{l}\text { Yes } \\
\text { No }\end{array}$ & $\begin{array}{c}\text { Yes } \\
\text { Yes }\end{array}$ & $\begin{array}{c}\text { Yes } \\
\text { Yes } \\
\text { Yes }\end{array}$ & $\begin{array}{c}\text { Yes } \\
\text { Yess }\end{array}$ & $\begin{array}{c}\text { No } \\
\text { Yes }\end{array}$ & 7 \\
\hline Yes & Yes & Yes & No & Yes & Yes & No & Yes & 6 \\
\hline 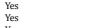 & $\begin{array}{c}\text { Kes } \\
\text { No } \\
\text { No }\end{array}$ & 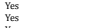 & $\begin{array}{c}\substack{\text { Yes } \\
\text { Yes }} \\
-\end{array}$ & $\begin{array}{c}\text { Yes } \\
\text { Yes } \\
\text { ves }\end{array}$ & $\begin{array}{c}\text { No } \\
\text { Yes }\end{array}$ & $\begin{array}{c}\text { No } \\
\text { Yes }\end{array}$ & $\begin{array}{l}\text { Yes } \\
\text { No } \\
\text { No }\end{array}$ & $\begin{array}{l}6 \\
6\end{array}$ \\
\hline $\begin{array}{l}\text { Yes } \\
\text { ves } \\
\text { Yes }\end{array}$ & $\begin{array}{l}\text { Yyes } \\
\text { Yyes } \\
\text { Yyes }\end{array}$ & $\begin{array}{l}\text { Yes } \\
\text { Yes } \\
\text { Yes }\end{array}$ & $\begin{array}{l}\text { YYes } \\
\text { No } \\
\text { Yys }\end{array}$ & $\begin{array}{l}\text { Yes } \\
\text { Yes } \\
\text { Yyes }\end{array}$ & $\begin{array}{l}\text { YYes } \\
\text { Yes } \\
\text { Yys }\end{array}$ & $\begin{array}{c}\text { Yes } \\
\text { Yes } \\
\text { ves }\end{array}$ & $\begin{array}{l}\text { Yes } \\
\text { No } \\
\text { Vos }\end{array}$ & $\begin{array}{l}8 \\
6 \\
8\end{array}$ \\
\hline 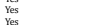 & $\substack{\text { Yes } \\
\text { Yes }}$ & 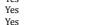 & $\begin{array}{c}\text { Yes } \\
\text { Yes } \\
\text { Yes }\end{array}$ & $\begin{array}{c}\text { Yes } \\
\text { Yes } \\
\text { Yes }\end{array}$ & $\begin{array}{c}\text { Yes } \\
\text { Yes } \\
\text { Yes }\end{array}$ & $\begin{array}{c}\text { Yes } \\
\text { Yos } \\
\text { No }\end{array}$ & 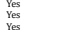 & $\begin{array}{l}8 \\
8 \\
7\end{array}$ \\
\hline Yes & Yes & № & No & Yes & Yes & Yes & Yes & 6 \\
\hline 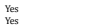 & $\substack{\text { Yes } \\
\text { ves }}$ & $\begin{array}{l}\text { Yes } \\
\text { yes } \\
\text { yes }\end{array}$ & $\begin{array}{c}\text { Yes } \\
\text { Yes } \\
\text { es }\end{array}$ & 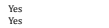 & $\begin{array}{c}\text { Yes } \\
\text { Yes } \\
\text { es }\end{array}$ & $\begin{array}{c}\text { Yes } \\
\text { No }\end{array}$ & 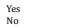 & $\begin{array}{l}8 \\
6 \\
6\end{array}$ \\
\hline $\begin{array}{l}\text { Ves } \\
\text { ves } \\
\text { ves }\end{array}$ & 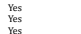 & $\begin{array}{l}\text { Yes } \\
\text { Yes } \\
\text { Yes }\end{array}$ & $\begin{array}{l}\text { Yes } \\
\text { Yes } \\
\text { Yos }\end{array}$ & $\begin{array}{c}\text { Yes } \\
\text { Yes } \\
\text { Yyes }\end{array}$ & $\begin{array}{l}\text { Yes } \\
\text { No } \\
\text { Nose }\end{array}$ & $\begin{array}{l}\text { No } \\
\text { No } \\
\text { Yose }\end{array}$ & $\begin{array}{l}\text { No } \\
\text { No } \\
\text { No }\end{array}$ & 5 \\
\hline 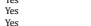 & 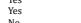 & $\begin{array}{l}\text { Pes } \\
\text { Yes } \\
\text { Yos }\end{array}$ & $\begin{array}{l}\text { No } \\
\text { No } \\
\text { Nots }\end{array}$ & $\begin{array}{c}\text { res } \\
\text { Yes } \\
\text { yes }\end{array}$ & $\begin{array}{c}\text { pes } \\
\text { Yes } \\
\text { Yos }\end{array}$ & $\begin{array}{l}\text { res } \\
\text { yes } \\
\text { Yos }\end{array}$ & $\begin{array}{l}\text { No } \\
\text { Yes } \\
\text { Yos }\end{array}$ & ${ }_{7}^{6}$ \\
\hline 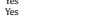 & & $\begin{array}{l}\text { Yes } \\
\text { Yes } \\
\text { Yos }\end{array}$ & $\begin{array}{l}\text { No } \\
\text { Yes }\end{array}$ & $\begin{array}{l}\text { Yes } \\
\text { Yes }\end{array}$ & $\begin{array}{l}\text { Yes } \\
\text { Yes }\end{array}$ & $\begin{array}{l}\text { Yyes } \\
\text { Yes }\end{array}$ & $\begin{array}{l}\text { Yes } \\
\text { No }\end{array}$ & ${ }_{7}^{6}$ \\
\hline Yes & Yes & № & No & Yes & Yes & Yes & No & 5 \\
\hline 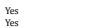 & $\begin{array}{c}\text { Yes } \\
\text { No } \\
\text { Nots }\end{array}$ & 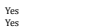 & $\begin{array}{c}\text { Yes } \\
\text { Yes }\end{array}$ & 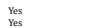 & $\begin{array}{c}\text { Yes } \\
\text { Yes }\end{array}$ & $\begin{array}{c}\text { Yes } \\
\text { No }\end{array}$ & $\begin{array}{l}\text { Yes } \\
\text { Yes } \\
\text { Yes }\end{array}$ & $\begin{array}{l}8 \\
6 \\
6\end{array}$ \\
\hline $\begin{array}{l}\text { Yes } \\
\text { ves } \\
\text { les }\end{array}$ & 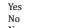 & 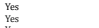 & $\begin{array}{l}\text { No } \\
\text { Yes }\end{array}$ & $\begin{array}{l}\text { Yes } \\
\text { Yes }\end{array}$ & $\begin{array}{l}\text { Yes } \\
\text { Yes }\end{array}$ & $\begin{array}{l}\text { Yes } \\
\text { Yes }\end{array}$ & $\begin{array}{l}\text { No } \\
\text { Yes }\end{array}$ & $\begin{array}{l}6 \\
7\end{array}$ \\
\hline Yes & Yes & Yes & No & $\begin{array}{c}\text { res } \\
\text { Yes }\end{array}$ & $\begin{array}{l}\text { Pres } \\
\text { Yes }\end{array}$ & $\begin{array}{l}\text { res } \\
\text { No }\end{array}$ & $\begin{array}{l}\text { Yes } \\
\text { Yes }\end{array}$ & 6 \\
\hline Yes & № & № & № & Yes & Yes & Yes & № & 4 \\
\hline Yes & Yes & № & № & Yes & Yes & Yes & Yes & 6 \\
\hline 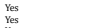 & 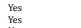 & $\begin{array}{l}\text { Yes } \\
\text { Yes } \\
\text { yes }\end{array}$ & $\begin{array}{c}\text { Yes } \\
\text { Yes }\end{array}$ & $\begin{array}{c}\text { Yes } \\
\text { Yes }\end{array}$ & $\begin{array}{c}\text { Yyes } \\
\text { Yes }\end{array}$ & $\begin{array}{c}\text { Yes } \\
\text { No }\end{array}$ & 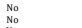 & ${ }_{6}^{7}$ \\
\hline $\begin{array}{l}\text { Yes } \\
\text { Yes } \\
\text { Kes }\end{array}$ & $\begin{array}{c}\text { No } \\
\text { kes }\end{array}$ & $\begin{array}{l}\text { Yes } \\
\text { Yes } \\
\text { Yos }\end{array}$ & $\begin{array}{l}\text { Yyes } \\
\text { Yes }\end{array}$ & $\begin{array}{c}\text { Yyes } \\
\text { Yes }\end{array}$ & $\begin{array}{c}\text { No } \\
\text { Yes }\end{array}$ & $\begin{array}{c}\text { Yes } \\
\text { Yes } \\
\text { Yos }\end{array}$ & $\begin{array}{l}\text { Yes } \\
\text { Yes }\end{array}$ & ${ }_{8}^{7}$ \\
\hline Yes & Yes & № & No & Yes & № & Yes & Yes & 5 \\
\hline Yes & Yes & Yes & Yes & Yes & Yes & No & № & 6 \\
\hline Yes & Yes & Yes & No & Yes & Yes & Yes & № & 6 \\
\hline Yes & Yes & Yes & Yes & Yes & Yes & Yes & No & 7 \\
\hline $\begin{array}{l}\text { Yes } \\
\text { Yes } \\
\text { ves }\end{array}$ & 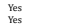 & $\begin{array}{l}\text { Yes } \\
\text { Yes } \\
\text { ves }\end{array}$ & $\begin{array}{c}\text { No } \\
\text { Yes }\end{array}$ & $\begin{array}{c}\text { Yes } \\
\text { Yes }\end{array}$ & $\begin{array}{c}\text { Yes } \\
\text { No } \\
\text { No }\end{array}$ & $\begin{array}{c}\text { Yes } \\
\text { Yes } \\
\text { Yos }\end{array}$ & $\begin{array}{l}\text { Yes } \\
\text { Yes } \\
\text { Yos }\end{array}$ & 7 \\
\hline $\begin{array}{l}\text { Yes } \\
\text { Ves } \\
\text { Vese }\end{array}$ & $\begin{array}{c}\text { Yes } \\
\text { Yes } \\
\text { Yes }\end{array}$ & $\begin{array}{l}\text { Yes } \\
\text { Yes } \\
\text { Yes }\end{array}$ & $\begin{array}{l}\text { Yes } \\
\text { No } \\
\text { Nose }\end{array}$ & $\begin{array}{c}\text { Yes } \\
\text { Yes } \\
\text { Yos }\end{array}$ & $\begin{array}{l}\text { Yes } \\
\text { No } \\
\text { Yos }\end{array}$ & $\begin{array}{l}\text { No } \\
\text { No }\end{array}$ & $\begin{array}{l}\text { Yes } \\
\text { Yes } \\
\text { ess }\end{array}$ & $\begin{array}{l}7 \\
5 \\
77\end{array}$ \\
\hline Yes & 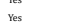 & 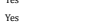 & No & $\begin{array}{l}\text { Pres } \\
\text { Yes }\end{array}$ & $\begin{array}{l}\text { Pes } \\
\text { No }\end{array}$ & $\begin{array}{l}\text { Nos } \\
\text { Yes }\end{array}$ & No & 5 \\
\hline $\begin{array}{l}\text { Yes } \\
\text { Yes }\end{array}$ & $\begin{array}{l}\text { Yes } \\
\text { Yes } \\
\text { Yes }\end{array}$ & $\begin{array}{l}\text { Yess } \\
\text { Ves }\end{array}$ & $\begin{array}{l}\text { Yes } \\
\text { Yes }\end{array}$ & $\begin{array}{l}\text { Yes } \\
\text { Yes }\end{array}$ & $\begin{array}{l}\text { Yes } \\
\text { Yes }\end{array}$ & $\begin{array}{l}\text { Yes } \\
\text { Yes }\end{array}$ & $\begin{array}{l}\text { Yes } \\
\text { No } \\
\text { No }\end{array}$ & 7 \\
\hline
\end{tabular}

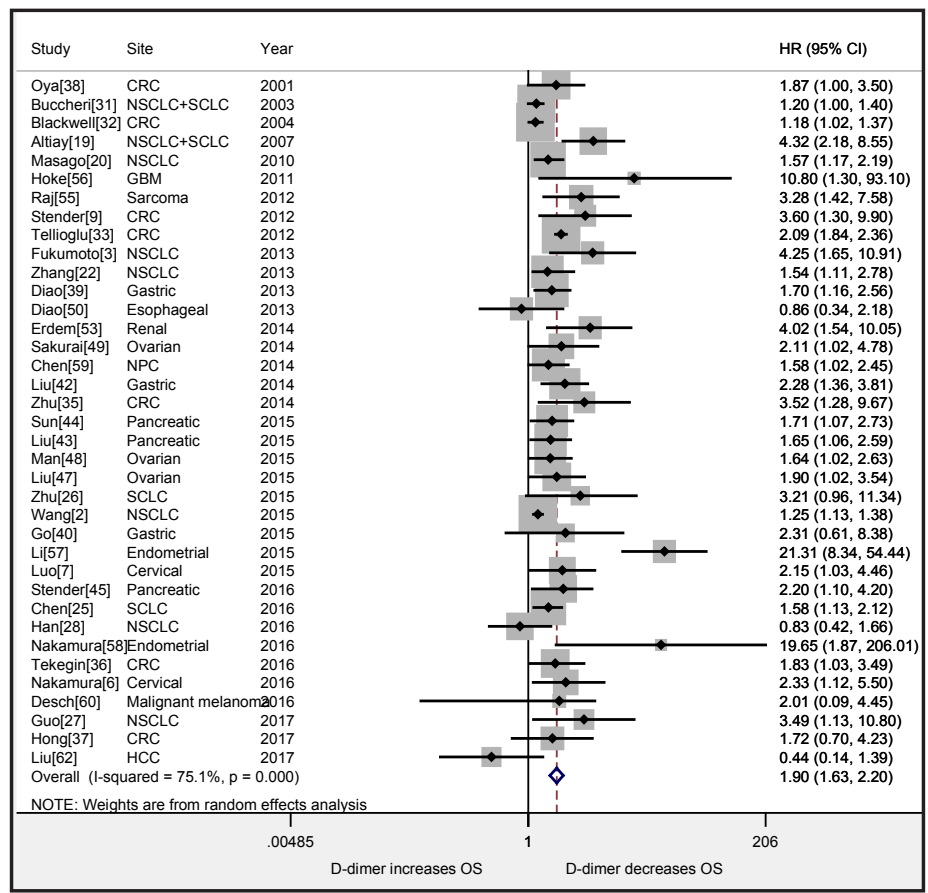

Fig. 2. Forest plots of hazard ratios for overall survival. 
Fig. 3. Forest plots displaying pooled hazard ratios (HRs) for overall survival according to cancer site subgroups.

of colorectal cancer $\left(\mathrm{I}^{2}=84.3, \mathrm{P}<\right.$ $0.001)$, lung cancer $\left(\mathrm{I}^{2}=69.3, \mathrm{P}<\right.$ $0.001)$ and other cancer $\left(I^{2}=62.5, P\right.$ $=0.014)$. As for subgroup analyses by disease stage, we found there exists a significant correlation between high D-dimer value and poor overall OS in patients with non-metastatic disease (pooled $\mathrm{HR}=1.70 ; 95 \% \mathrm{CI}=1.16-2.47 ; \mathrm{P}$ $=0.006)$ and metastatic disease (pooled $\mathrm{HR}=1.60 ; 95 \% \mathrm{CI}=$ 1.15-2.23; $\mathrm{P}=0.006)$. The HR for the mixed group including both metastatic and non-metastatic diseases was $2.10(95 \% \mathrm{CI}=$ 1.71-2.60; $\mathrm{P}<0.001)$. There are two main detection methods (ITM and Latex test) with various cut-off values used in the studies (from $130 \mathrm{ng} / \mathrm{ml}$ to $2000 \mathrm{ng} /$ $\mathrm{ml}$ ). Therefore, we divided those articles into different subgroups and found that patients with a high plasma D-dimer level have a worse prognosis than those with a low D-dimer level in all groups. The pooled HR >

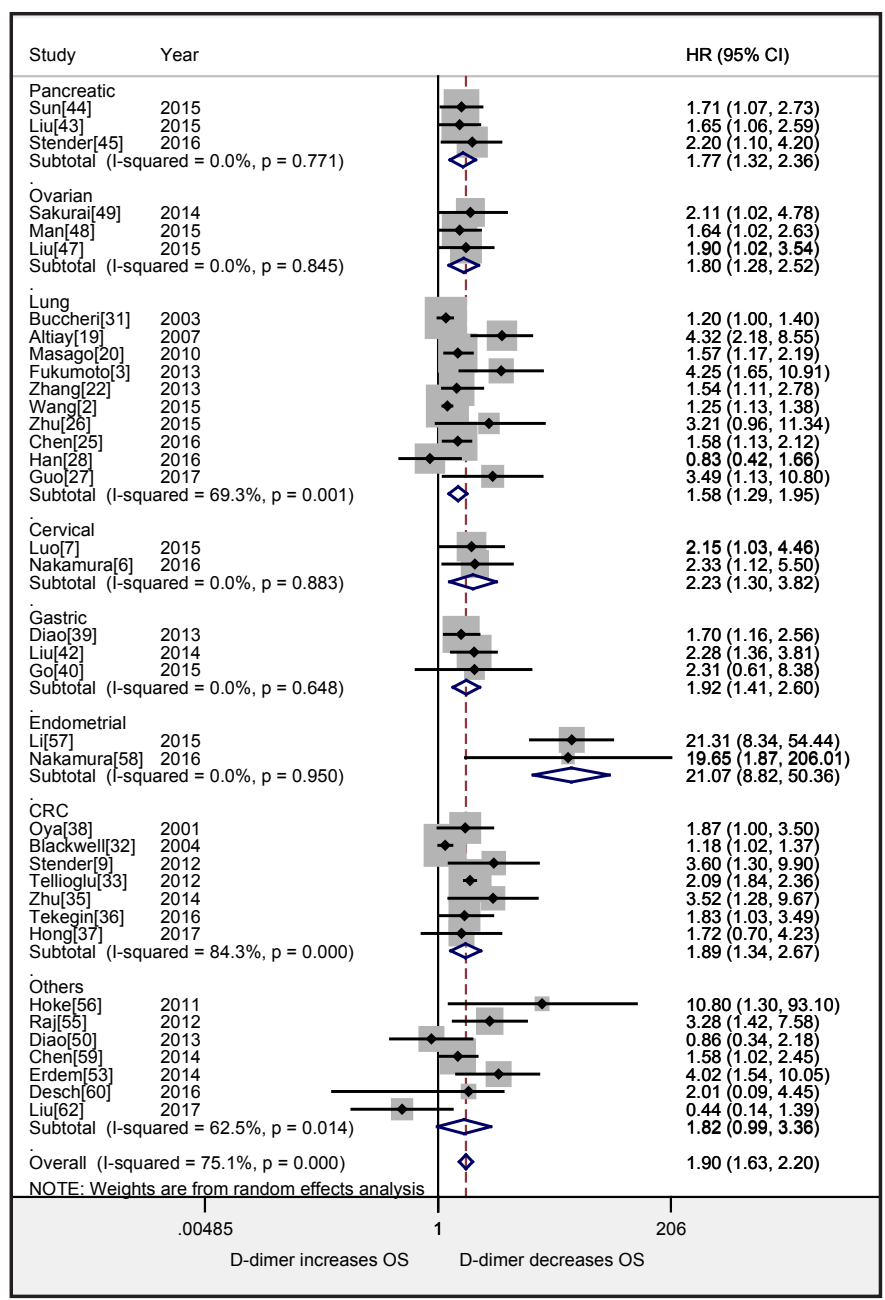
1 were consistently observed in subgroups analyses stratified by ethnicity (Non-Asian or Asian) and study design (retrospective or prospective), which provide robust evidence of plasma D-dimer levels in cancer patients could predict their prognosis regardless of ethnicity and study design (Table 3).

Sensitivity analysis using a "one-study removed" model was conducted to testify the stability of our result. As shown in Fig. 4A, the observed effect size (pooled multivariableadjusted HR) of OS was not significantly affected by removing a single study each time. Meta-regression showed that our results were not statistically impacted by patients' ethnicity $(p=0.671)$, cut-off points $(p=0.217)$, clinical setting $(p=0.998)$ and study design $(\mathrm{p}=0.967)$. Publication bias was found by visual inspection of the funnel plot Fig. 5D and confirmed by Begg's test and Egger's tests (all $\mathrm{P}<0.05$ ). However, using the "Trim and Fill" method to adjust

Table 3. Pooled multivariable-adjusted hazard ratios for OS according to subgroup analyses. CI = confidence interval; $\mathrm{HR}=$ hazard ratio; ITM = immunoturbidimetry; OS = overall survival; $\mathrm{PD}=\mathrm{P}$ for subgroup difference;

\begin{tabular}{lllllll}
\hline Subgroups & Studies & Pooled HR & $95 \% \mathrm{Cl}$ & P value & Heterogeneity $\left(1^{2}\right)$ & $\begin{array}{l}\text { Meta-regression } \\
\text { P-value }\end{array}$ \\
\hline $\begin{array}{l}\text { Disease stage } \\
\quad\end{array}$ & & & & & & 0.998 \\
$\quad$ Non-metastatic & 7 & 1.70 & $1.16-2.47$ & 0.006 & $50.2 \%$ & \\
$\quad$ Mixed & 25 & 2.10 & $1.71-2.60$ & $<0.001$ & $68.2 \%$ & \\
Metastatic & 5 & 1.60 & $1.15-2.23$ & 0.006 & $92.4 \%$ & \\
& & & & & & 0.967 \\
Type of data collection & & & & & & \\
$\quad$ Retrospective & 22 & 1.91 & $1.53-2.37$ & $<0.001$ & $67.8 \%$ & \\
$\quad$ Prospective & 15 & 1.93 & $1.54-2.43$ & $<0.001$ & $82.3 \%$ & \\
& & & & & & \\
Ethnicity & & & & & & 0.671 \\
Asian & 30 & 1.97 & $1.64-2.37$ & $<0.001$ & $68.8 \%$ & \\
Non-Asian & 7 & 1.76 & $1.27-2.45$ & 0.001 & $88.2 \%$ & \\
& & & & & & \\
Cut-off points & & & & & & 0.217 \\
ITM & & & & & & \\
$\leq 299$ & 2 & 2.14 & $1.03-4.42$ & 0.041 & $0 \%$ & \\
$300-499$ & 3 & 1.65 & $1.24-2.21$ & 0.001 & $0 \%$ & \\
$500-999$ & 8 & 1.51 & $1.26-1.80$ & $<0.001$ & $29.4 \%$ & \\
$\geq 1000$ & 4 & 1.32 & $1.03-1.70$ & 0.029 & $24.9 \%$ & \\
Latex Test & & & & & & \\
$300-499$ & 1 & 3.60 & $1.30-9.93$ & 0.013 & -- & \\
$500-999$ & 1 & 1.87 & $1.00-3.50$ & 0.049 & -- & \\
$\geq 1000$ & 7 & 4.50 & $2.15-9.93$ & $<0.001$ & $72.6 \%$ & \\
\hline & & & & & &
\end{tabular}




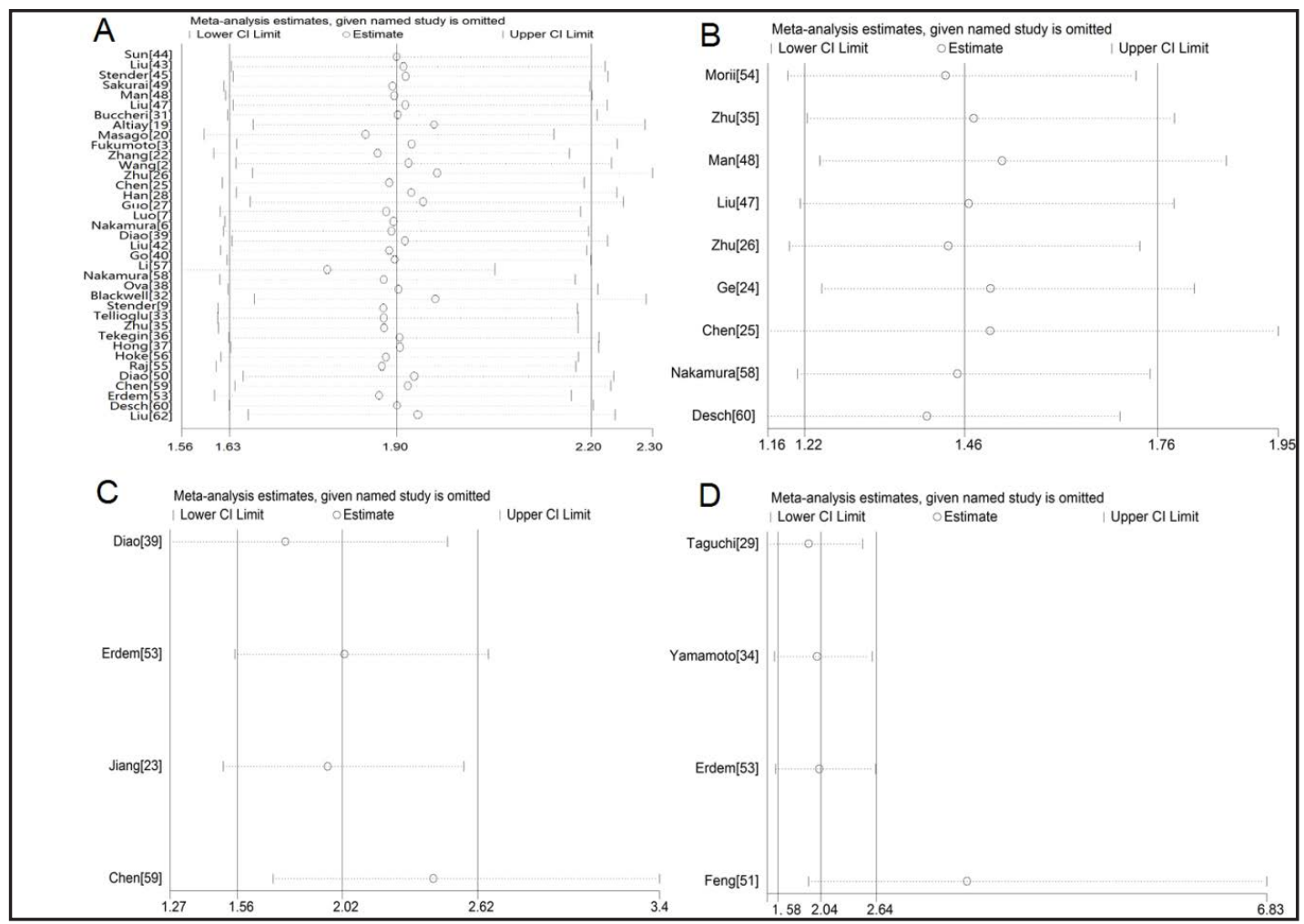

Fig. 4. Sensitivity analysis using a "one-study removed" model shows that the pooled effect size for (A) overall survival; (B) progression-free survival; (C) disease-free survival; (D) cancer-specific survival is not significantly affected by removing a single study each time, respectively.

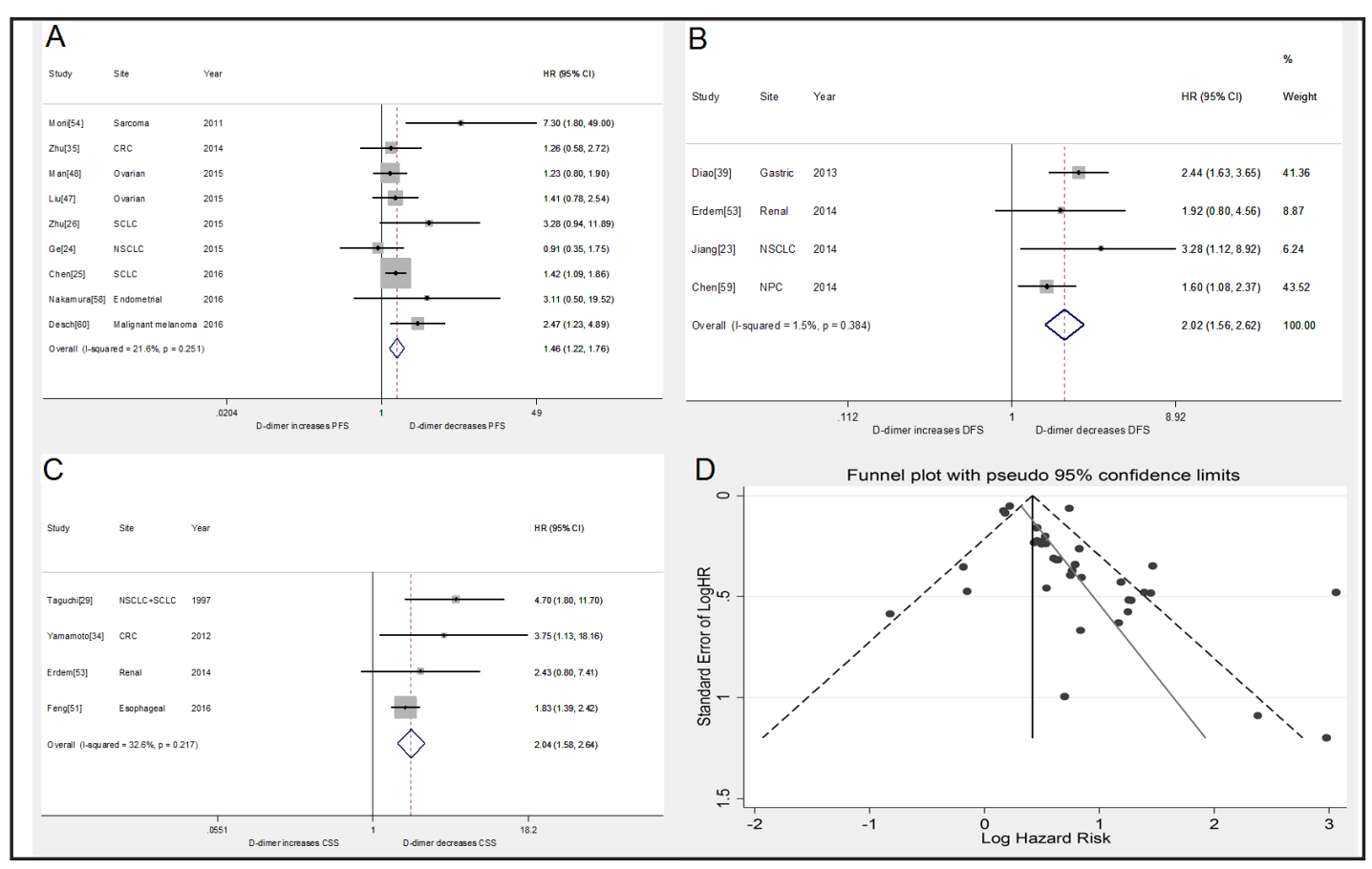

Fig. 5. Forest plots of hazard ratios for (A) progression-free survival; (B) disease-free survival; (C) cancerspecific survival. D. Funnel plot of publication bias in the meta-analysis. Funnel plot of log HR (horizontal axis) against its standard error (vertical axis) for each individual study (represented by one circle). The vertical line represents the pooled effect estimate. 


\section{Cellular Physiology Cell Physiol Biochem 2018;45:1663-1676 \begin{tabular}{ll|l} 
DOI: 10.1159/000487734 & (0) 2018 The Author(s). Published by S. Karger AG, Basel \\
and Biochemistry & Published online: February 27, 2018 www.karger.com/cpb
\end{tabular} \\ Li et al.: Prognositc Role of D-Dimer in Solid Tumors: a Meta-Analysis}

for publication bias, the corrected pooled effect size remained statistically significant (HR $=1.521,95 \% \mathrm{CI}=1.305-1.774 ; \mathrm{P}<0.001$ under the random-effects model). Thereby the reliability of our results could be confirmed.

A separate meta-analysis of twenty-two studies containing 5320 patients based on univariate HRs for OS showed that D-dimer level was statistically significant correlated with worse OS (pooled unadjusted HR $=1.88 ; 95 \% \mathrm{CI}=1.67-2.13 ; \mathrm{P}<0.001$ ) with high heterogeneity $\left(\mathrm{I}^{2}=77.8 ; \mathrm{P}<0.001\right)$. The result was consistent with pooled multivariableadjusted HR analysis for OS.

Secondary outcome: Progression-free survival, Disease-free survival and Cancer-specific survival

A total of nine studies, comprising 1231 patients, provided pooled multivariableadjusted HRs data for PFS. Overall, elevated D-dimer was significantly associated with worse PFS (HR = 1.46, 95\% CI = 1.22-1.76; $\mathrm{P}<0.001)$ using a fixed-effects model $\left(\mathrm{I}^{2}=21.6 ; \mathrm{P}=\right.$ 0.251 ) Fig. 5A. Five studies, including 2359 patients, provided data for the analysis of HRs for DFS. Of those, four studies containing 1891 patients, provided multivariable-adjusted HRs for DFS and were included in our analyses. As shown in Fig. 5B, high D-dimer level was significantly associated with poor DFS in a fixed-effects model (pooled multivariableadjusted $\mathrm{HR}=2.02,95 \% \mathrm{CI}=1.56-2.62 ; \mathrm{P}<0.001$ ) with insignificant heterogeneity among studies $\left(\mathrm{I}^{2}=1.5 \%, \mathrm{P}=0.384\right)$. We found five articles containing data for the analysis of HRs for CSS, four of them (comprising a total of 577 patients) provided multivariable-adjusted HRs and therefore included in our analyses. As shown in Fig. 5C, the pooled HRs for CSS was $2.04(95 \% \mathrm{CI}=1.58-2.64 ; \mathrm{P}<0.001)$. Insignificant heterogeneity across studies was observed $\left(\mathrm{I}^{2}=32.6 ; \mathrm{P}=0.217\right)$. Sensitivity analysis was performed and we found that the observed effect size (pooled multivariable-adjusted HR) of PFS Fig. 4B and DFS Fig. 4C was not significantly influenced by removing a single study each time. However, the pooled HR for CSS increased dramatically when we removed Feng et al . [51] Fig. 4D.

\section{Discussion}

Our study provided strong evidence that an elevated pretreatment plasma D-dimer is associated with unfavorable overall survival among various cancer subgroups and across disease stages. The pooled HRs on OS was 1.90 (95\% CI: 1.63 - 2.20). By analyzing the subgroup stratified by cancer type, we found that the magnitude effect of D-dimer on OS appeared to perform its best predicted effect on cancer patient's prognosis among gynecological tumors. The highest effect was seen in endometrial cancer, followed by cervical cancer and gastric cancer. Unfavorable prognosis effect of high D-dimer remained substantial in different clinical cancer settings, different ethnicities, different detection methods with various cutoff values. The prognosis impact of a high plasma D-dimer before treatment on secondary endpoints (DFS, CSS and PFS) was consistent with OS, all pooled HRs $>1$ indicating D-dimer is an unfavorable prognostic factor to cancer patients.

D-dimer is a universally available, routinely measured and simple reproducible molecular marker. Plasma D-dimer level is currently being routinely measured prior to operation. To our knowledge, this is the first article that combines all solid tumors prognostic data together to explore the prognosis value of pretreatment plasma D-dimer. The result in our analysis may be different from other meta-analyses [63], which is partly attributed to different inclusion criteria and different research interests. Unlike other meta-analyses, we only included pretreatment D-dimer data as our analysis objects, the number of eligible studies is small but the quality and reliability of our analysis were guaranteed. Furthermore, the current cancer prognostic evaluation system has its own drawbacks that patients with the same TNM stage often have different survival outcome. D-dimer may serve as a complementary indicator that could help to improve prognosis estimation. In the current meta-analysis, we only applied HR instead of OR or RR to estimate the prognostic value, because the latter two dichotomous 
measures are either unreliable or difficult to interpret. Meanwhile, since D-dimer level could be influenced by many factors such as age, sex, tumor stage (For example, plasma D-dimer baseline level elevates with age), we adopted the multivariable-adjusted HRs to minimize the interference of confounding factors instead of univariate analyses as the main data source for this meta-analysis, for the latter may overestimate effect sizes. The robustness of results on the prognostic role of D-dimer (OS, PFS and DFS) was confirmed by sensitivity analysis, demonstrating the pooled HR was not significantly affected by individual. There are only 4 studies provided pooled multivariable-adjusted HR data for CSS, and the samples size of one study is larger than the total of the rest three, therefore we consider the quality of our result is not high and more studies are still needed to elucidated its clinical significance.

The mechanism of how D-dimer affect prognosis is still unknown to this day. Recent studies suggested that there exists a potential relationship between coagulation, fibrinolysis system, and tumor prognosis. Perisanidis et al. performed a meta-anlysis containing 52 studies with 15371 participants [64]. concluded that elevated pretreatment plasma fibrinogen significantly correlates with decreased survival in patients with solid tumors and came to a conclusion that fibrinogen may play a critical role in tumor progression, including epithelial-mesenchymal transition (EMT), migration, cell proliferation, angiogenesis and hematogenous metastasis [65-67]. D-dimer is known to be a degradation product of fibrin, some researchers believed D-dimer affect cancer patients' prognosis through the formation of VTE. VTE is a common complication of cancer $[68,69]$. Overall, the risk of VTE in patients with cancer is as high as 7\%, presumably due to the prothrombotic effect of malignant tumors and treatment-related risk factors such as immobilization, drugs, and surgical intervention [70]. However, Ay et al. [21] reported that according to their large scale prospective study data, the association of D-dimer and VTE with an unfavorable prognosis of cancer was independent of each other in solid tumors. They explained this finding with a hypothesis that abnormal elevated D-dimer may be a product of a reaction downstream from the extrinsic pathway of the coagulation cascade. Tissue factor (TF) is a key element in the initiation of the extrinsic pathway and is considered to play an important role in cancer metastasis and progression. Thus, elevated D-dimer may be a result of abnormally activated TF.

Poor prognosis of cancer patients always coincides with metastasis, a process which involves multiple tumor-host interactions. Metastatic cancer cells must leave the primary tumor, migrate into the lymphovascular system, and set up a new blood supply system. Fibrin remodeling is almost involved in all steps of metastasis and has been proven to play a crucial role in new vessel formation [74, 75]. Therefore, Blackwell et al. [76] declared that increased D-dimer levels in cancer patients may be an outcome of increased levels of fibrinolytic activity. Clinical trials about treating cancer patients with anticoagulation therapy have been carried out and reports have shown that anti-thrombotic agents, such as warfarin and low molecular weight heparin, are effective in the prevention and treatment of haematogenous metastasis as well as in the prolongation of survival [77-79]. Further studies are still needed to elucidate the biological mechanism of how high D-dimer affects the progression of malignancy as well as the treatment efficiency of anticoagulation therapy in cancer patients.

Certain limitations should be taken into consideration when interpreting study findings. Firstly, in this analysis, only 71.4\% (35/49) articles excluded patients with hematologic diseases or have received anticoagulation therapy. Although most of studies involved in our meta-analysis declared that high D-dimer concentration is an independent marker for cancer prognosis, it still indicates a high risk of VTE. According to Li et al. [57], subclinical VTE prior to surgery exists in at least $10 \%$ of patients with endometrial cancer, those undetectable VTE may put efforts on tumor prognosis. Secondly, the data we used are all extracted from published articles or directly provided by authors instead of individual participant. We also included studies provided low quality data with short duration of follow-up. Third, high heterogeneity was observed across studies since our evaluation was based on factors such as different tumor types, disease stages, measurement methods and study designs. However, if we sort these data into different subgroup analyses, a more homogeneous data was observed. Lastly, we only included studies reporting hazard ratios, therefore some studies reporting on 
the prognostic value of D-dimer were excluded (Articles only reported odds ratio for death which may introduce selection bias). Meanwhile, we only included published articles in this study, there may exist some negative result studies that were never been published. These two limitations may cause selection bias. However, the corrected pooled effect size remained statistically significant after being adjusted by "Trim and Fill" method, indicating our results could be trusted.

\section{Conclusion}

In summary, our meta-analysis provided evidence that elevated pretreatment plasma D-dimer is associated with adverse survival among patients with different types of solid tumors. Further observational and intervention studies are still needed to determine whether plasma D-dimer could be incorporated in cancer staging system. Additionally, more research should be conducted to elucidate the relationship between high D-dimer and tumor progression.

\section{Abbreviations}

ASCO (American Society of Clinical Oncology); CI (confidence interval); CSS (cancerspecific survival); DFS (disease-free survival); DIC (disseminated intravascular coagulation); DVT (deep venous thrombosis); EMT (epithelial-mesenchymal transition); OS (overall survival); PE (pulmonary embolis); PFS (progression-free survival); PRISMA (Systematic Reviews and Meta-Analyses); STROBE (Study OBservational studies in Epidemiology); TF (tissue factor).

\section{Acknowledgements}

The meta-analysis does not need ethics approval and consent to participate.

The study was sponsored by China Scholarship Council.

Experimental design: Dongmin Chang, Wenhan Li; Study selection: Yongchun Song, Hao Zhang; Data analysis: Dongmin Chang, Yao Tang, Wenhan Li, Ming Ni, Xin Xie, Qingnuo Zeng, Bin Hou; Article writing: Wenhan Li, Navard Sisliyan, Szu Hao Chen.

\section{Disclosure Statement}

No conflict of interest exists.

\section{References}

1 Komurcuoglu B, Ulusoy S, Gayaf M, Guler A, Ozden E: Prognostic value of plasma D-dimer levels in lung carcinoma. Tumori 2011;97:743-748.

-2 Wang Y, Wang Z: Predictive value of plasma D-dimer levels in patients with advanced non-small-cell lung cancer. Onco Targets Ther 2015;8:805-808.

- Fukumoto K, Taniguchi T, Usami N, Kawaguchi K, Fukui T, Ishiguro F, Nakamura S, Yokoi K: Preoperative plasma D-dimer level is an independent prognostic factor in patients with completely resected non-small cell lung cancer. Surg Today 2015;45:63-67.

4 Nakashima J, Tachibana M, Ueno M, Baba S, Tazaki H: Tumor necrosis factor and coagulopathy in patients with prostate cancer. Cancer Res 1995;55:4881-4885. 


\section{Cellular Physiology Cell Physiol Biochem 2018;45:1663-1676 \begin{tabular}{l|l|l|l} 
DOI: 10.1159/000487734 2018 The Author(s). Published by S. Karger AG, Basel & (0)
\end{tabular} and Biochemistry Published online: February 27, 2018 www.karger.com/cpb}

Li et al.: Prognositc Role of D-Dimer in Solid Tumors: a Meta-Analysis

5 Okajima K, Kohno I, Tsuruta J, Okabe H, Takatsuki K, Binder BR: Direct evidence for systemic fibrinogenolysis in a patient with metastatic prostatic cancer. Thromb Res 1992;66:717-727.

6 Nakamura K, Nakayama K, Ishikawa M, Katagiri H, Minamoto T, Ishibashi T, Ishikawa N, Sato E, Sanuki K, Yamashita H, Komatsu-Fujii T, Kyo S: High Pre-treatment Plasma D-Dimer Level as a Potential Prognostic Biomarker for Cervical Carcinoma. Anticancer Res 2016;36:2933-2938.

7 Luo YL, Chi PD, Zheng X, Zhang L, Wang XP, Chen H: Preoperative D-dimers as an independent prognostic marker in cervical carcinoma. Tumour Biol 2015;36:8903-8911.

-8 Ferroni P, Roselli M, Portarena I, Formica V, Riondino S, F LAF, Costarelli L, Melino A, Massimiani G, Cavaliere F, Palmirotta R, Guadagni F: Plasma plasminogen activator inhibitor-1 (PAI-1) levels in breast cancer - relationship with clinical outcome. Anticancer Res 2014;34:1153-1161.

-9 Stender MT, Larsen TB, Sorensen HT, Thorlacius-Ussing 0: Preoperative plasma D-dimer predicts 1-year survival in colorectal cancer patients with absence of venous thromboembolism (VTE): a prospective clinical cohort study. J Thromb Haemost 2012;10:2027-2031.

10 Aliustaoglu M, Yumuk PF, Gumus M, Ekenel M, Bolukbas F, Bolukbas C, Mutlu N, Basaran G, Avsar E, Turhal NS: D-dimer--can it be a marker for malignant gastric lesions? Acta Oncol 2004;43:770-771.

11 Palumbo JS, Kombrinck KW, Drew AF, Grimes TS, Kiser JH, Degen JL, Bugge TH: Fibrinogen is an important determinant of the metastatic potential of circulating tumor cells. Blood 2000;96:3302-3309.

12 Thachil J, Fitzmaurice DA, Toh CH: Appropriate use of D-dimer in hospital patients. Am J Med 2010;123:1719.

13 Spring JL, Winkler A, Levy JH: The influence of various patient characteristics on D-dimer concentration in critically ill patients and its role as a prognostic indicator in the intensive care unit setting. Clin Lab Med 2014;34:675-686.

14 Moher D, Liberati A, Tetzlaff J, Altman DG: Preferred reporting items for systematic reviews and metaanalyses: the PRISMA statement. Int J Surg 2010;8:336-341.

15 von Elm E, Altman DG, Egger M, Pocock SJ, Gotzsche PC, Vandenbroucke JP: The Strengthening the Reporting of Observational Studies in Epidemiology (STROBE) Statement: guidelines for reporting observational studies. Int J Surg 2014;12:1495-1499.

16 Tierney JF, Stewart LA, Ghersi D, Burdett S, Sydes MR: Practical methods for incorporating summary timeto-event data into meta-analysis. Trials 2007;8:16.

17 Parmar MK, Torri V, Stewart L: Extracting summary statistics to perform meta-analyses of the published literature for survival endpoints. Stat Med 1998;17:2815-2834.

18 Duval S, Tweedie R: Trim and fill: A simple funnel-plot-based method of testing and adjusting for publication bias in meta-analysis. Biometrics 2000;56:455-463.

19 Altiay G, Ciftci A, Demir M, Kocak Z, Sut N, Tabakoglu E, Hatipoglu ON, Caglar T: High Plasma d-dimer Level is Associated with Decreased Survival in Patients with Lung Cancer. Clinical Oncology 2007;19:494-498.

20 Masago K, Fujita S, Mio T, Togashi Y, Kim YH, Hatachi Y, Fukuhara A, Irisa K, Sakamori Y, Mishima M: Clinical significance of the ratio between the alpha 2 plasmin inhibitor-plasmin complex and the thrombinantithrombin complex in advanced non-small cell lung cancer. Med Oncol 2011;28:351-356.

-21 Ay C, Dunkler D, Pirker R, Thaler J, Quehenberger P, Wagner O, Zielinski C, Pabinger I: High D-dimer levels are associated with poor prognosis in cancer patients. Haematologica 2012;97:1158-1164.

22 Zhang L, Ridgway LD, Wetzel MD, Ngo J, Yin W, Kumar D, Goodman JC, Groves MD, Marchetti D: The identification and characterization of breast cancer CTCs competent for brain metastasis. Sci Transl Med 2013;5:180ra148.

23 Jiang HG, Li J, Shi SB, Chen P, Ge LP, Jiang Q Tang XP: Value of fibrinogen and D-dimer in predicting recurrence and metastasis after radical surgery for non-small cell lung cancer. Med Oncol 2014;31:22.

24 Ge LP, Li J, Bao QL, Chen P, Jiang Q, Zhu LR: Prognostic and predictive value of plasma D-dimer in advanced non-small cell lung cancer patients undergoing first-line chemotherapy. Clin Transl Oncol 2015;17:57-64.

25 Chen Y, Yu H, Wu C, Li J, Jiao S, Hu Y, Tao H, Wu B, Li A: Prognostic value of plasma D-dimer levels in patients with small-cell lung cancer. Biomed Pharmacother 2016;81:210-217.

-26 Zhu LR, Li J, Chen P, Jiang Q, Tang XP: Clinical significance of plasma fibrinogen and D-dimer in predicting the chemotherapy efficacy and prognosis for small cell lung cancer patients. Clin Transl Oncol 2016;18:178-188. 


\section{Cellular Physiology Cell Physiol Biochem 2018;45:1663-1676

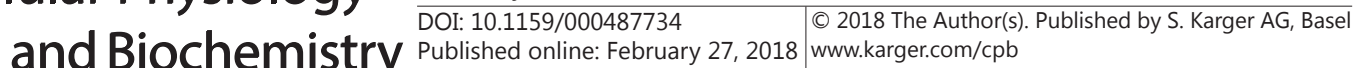

27 Guo R, Yang J, Liu X, Wu J, Chen Y: Increased von Willebrand factor over decreased ADAMTS-13 activity is associated with poor prognosis in patients with advanced non-small-cell lung cancer. J Clin Lab Anal 2017;10.1002/jcla.22219

28 Han Y, Wang J, Hong L, Sun L, Zhuang H, Sun B, Wang H, Zhang X, Ren X: Platelet-lymphocyte ratio is an independent prognostic factor in patients with ALK-positive non-small-cell lung cancer. Future Oncol 2017;13:51-61.

29 Taguchi O, Gabazza EC, Yasui H, Kobayashi T, Yoshida M, Kobayashi H: Prognostic significance of plasma D-dimer levels in patients with lung cancer. Thorax 1997;52:563-565.

-30 Ferrigno D, Buccheri G, Ricca I: Prognostic significance of blood coagulation tests in lung cancer. Eur Respir J 2001;17:667-673.

-31 Buccheri G, Torchio P, Ferrigno D: Plasma levels of D-dimer in lung carcinoma: clinical and prognostic significance. Cancer 2003;97:3044-3052.

-32 Blackwell K, Hurwitz H, Lieberman G, Novotny W, Snyder S, Dewhirst M, Greenberg C: Circulating D-dimer levels are better predictors of overall survival and disease progression than carcinoembryonic antigen levels in patients with metastatic colorectal carcinoma. Cancer 2004;101:77-82.

-33 Tellioglu G, Agcaoglu 0, Siperstein A, Berber E: Serum D-dimer as a prognostic marker in patients undergoing radiofrequency ablation of colorectal liver metastasis. J Invest Surg 2012;25:295-300.

34 Yamamoto M, Yoshinaga K, Matsuyama A, Iwasa T, Osoegawa A, Tsujita E, Yamashita Y, Tsutsui S, Ishida T: Plasma D-dimer level as a mortality predictor in patients with advanced or recurrent colorectal cancer. Oncology 2012;83:10-15.

-35 Zhu L, Liu B, Zhao Y, Liu L, Yang C, Yang Y, Zhong H: High levels of D-dimer correlated with disease status and poor prognosis of inoperable metastatic colorectal cancer patients treated with bevacizumab. J Cancer Res Ther 2014;10 Suppl:246-251.

-36 Tekesin K, Bayrak S, Esatoglu V, Ozdemir E, Ozel L, Melih Kara V: D-Dimer and Carcinoembryonic Antigen Levels: Useful Indicators for Predicting the Tumor Stage and Postoperative Survival. Gastroenterol Res Pract 2016;2016:4295029.

37 Hong T, Shen D, Chen X, Wu X, Hua D: Preoperative plasma fibrinogen, but not D-dimer might represent a prognostic factor in non-metastatic colorectal cancer: A prospective cohort study. Cancer Biomark 2017;19:103-111.

38 Oya M, Akiyama Y, Okuyama T, Ishikawa H: High preoperative plasma D-dimer level is associated with advanced tumor stage and short survival after curative resection in patients with colorectal cancer. Jpn J Clin Oncol 2001;31:388-394.

39 Diao D, Wang Z, Cheng Y, Zhang H, Guo Q, Song Y, Zhu K, Li K, Liu D, Dang C: D-dimer: not just an indicator of venous thrombosis but a predictor of asymptomatic hematogenous metastasis in gastric cancer patients. PLoS One 2014;9:e101125.

-40 Go SI, Lee MJ, Lee WS, Choi HJ, Lee US, Kim RB, Kang MH, Kim HG, Lee GW, Kang JH, Lee JH, Kim SJ: D-Dimer Can Serve as a Prognostic and Predictive Biomarker for Metastatic Gastric Cancer Treated by Chemotherapy. Medicine (Baltimore) 2015;94:e951.

41 Kanda M, Tanaka C, Kobayashi D, Mizuno A, Tanaka Y, Takami H, Iwata N, Hayashi M, Niwa Y, Yamada S, Fujii T, Sugimoto H, Murotani K, Fujiwara M, Kodera Y: Proposal of the Coagulation Score as a Predictor for Short-Term and Long-Term Outcomes of Patients with Resectable Gastric Cancer. Ann Surg Oncol 2017;24:502-509.

42 Liu L, Zhang X, Yan B, Gu Q, Zhang X, Jiao J, Sun D, Wang N, Yue X: Elevated plasma D-dimer levels correlate with long term survival of gastric cancer patients. PLoS One 2014;9:e90547.

43 Liu P, Zhu Y, Liu L: Elevated pretreatment plasma D-dimer levels and platelet counts predict poor prognosis in pancreatic adenocarcinoma. Onco Targets Ther 2015;8:1335-1340.

-44 Sun W, Ren H, Gao CT, Ma WD, Luo L, Liu Y, Jin P, Hao JH: Clinical and Prognostic Significance of Coagulation Assays in Pancreatic Cancer Patients With Absence of Venous Thromboembolism. Am J Clin Oncol 2015;38:550-556.

45 Stender MT, Larsen AC, Sall M, Thorlacius-Ussing 0: D-Dimer predicts prognosis and non-resectability in patients with pancreatic cancer: a prospective cohort study. Blood Coagul Fibrinolysis 2016;27:597-601.

46 Cao J, Fu Z, Gao L, Wang X, Cheng S, Wang X, Ren H: Evaluation of serum D-dimer, fibrinogen, and CA19-9 for postoperative monitoring and survival prediction in resectable pancreatic carcinoma. World J Surg Oncol 2017;15:48. 


\section{Cellular Physiology Cell Physiol Biochem 2018;45:1663-1676 and Biochemistry DOI: 10.1159/000487734 27, 2018 (O) 2018 The Author(s). Published by S. Karger AG, Basel

47 Liu P, Wang Y, Tong L, Xu Y, Zhang W, Guo Z, Ni H: Elevated preoperative plasma D-dimer level is a useful predictor of chemoresistance and poor disease outcome for serous ovarian cancer patients. Cancer Chemother Pharmacol 2015;76:1163-1171.

48 Man YN, Wang YN, Hao J, Liu X, Liu C, Zhu C, Wu XZ: Pretreatment plasma D-dimer, fibrinogen, and platelet levels significantly impact prognosis in patients with epithelial ovarian cancer independently of venous thromboembolism. Int J Gynecol Cancer 2015;25:24-32.

49 Sakurai M, Satoh T, Matsumoto K, Michikami H, Nakamura Y, Nakao S, Ochi H, Onuki M, Minaguchi T, Yoshikawa H: High Pretreatment Plasma D-dimer Levels Are Associated With Poor Prognosis in Patients With Ovarian Cancer Independently of Venous Thromboembolism and Tumor Extension. Int J Gynecol Cancer 2015;25:593-598.

50 Diao D, Zhu K, Wang Z, Cheng Y, Li K, Pei L, Dang C: Prognostic value of the D-dimer test in oesophageal cancer during the perioperative period. J Surg Oncol 2013;108:34-41.

-51 Feng JF, Yang X, Chen S, Zhao Q, Chen QX: Prognostic Value of Plasma D-dimer in Patients with Resectable Esophageal Squamous Cell Carcinoma in China. J Cancer 2016;7:1663-1667.

52 Zhang F, Chen Z, Wang P, Hu X, Gao Y, He J: Combination of platelet count and mean platelet volume (COPMPV) predicts postoperative prognosis in both resectable early and advanced stage esophageal squamous cell cancer patients. Tumour Biol 2016;37:9323-9331.

53 Erdem S, Amasyali AS, Aytac O, Onem K, Issever H, Sanli O: Increased preoperative levels of plasma fibrinogen and D dimer in patients with renal cell carcinoma is associated with poor survival and adverse tumor characteristics. Urol Oncol 2014;32:1031-1040.

54 Morii T, Mochizuki K, Tajima T, Ichimura S, Satomi K: D-dimer levels as a prognostic factor for determining oncological outcomes in musculoskeletal sarcoma. BMC Musculoskelet Disord 2011;12:250.

55 Raj SD, Zhou X, Bueso-Ramos CE, Ravi V, Patel S, Benjamin RS, Vadhan-Raj S: Prognostic significance of elevated D-dimer for survival in patients with sarcoma. Am J Clin Oncol 2012;35:462-467.

-56 Hoke M, Dieckmann K, Koppensteiner R, Schillinger M, Marosi C, Mlekusch W: Prognostic value of plasma d-dimer levels in patients with glioblastoma multiforme - Results from a pilot study. Wien Klin Wochenschr 2011;123:199-203.

57 Li J, Lin J, Luo Y, Kuang M, Liu Y: Multivariate Analysis of Prognostic Biomarkers in Surgically Treated Endometrial Cancer. PLoS One 2015;10:e0130640.

58 Nakamura K, Nakayama K, Ishikawa M, Katagiri H, Minamoto T, Ishibashi T, Ishikawa N, Sato E, Sanuki K, Yamashita H, Komatsu-Fujii T, Kyo S: High pretreatment plasma D-dimer levels are related to shorter overall survival in endometrial carcinoma. Eur J Obstet Gynecol Reprod Biol 2016;201:89-93.

59 Chen WH, Tang LQ, Wang FW, Li CP, Tian XP, Huang XX, Mai SJ, Liao YJ, Deng HX, Chen QY, Liu H, Zhang L, Guo SS, Liu LT, Yan SM, Li CF, Zhang JP, Liu Q Liu XW, Liu LZ, Mai HQ Zeng MS, Xie D: Elevated levels of plasma D-dimer predict a worse outcome in patients with nasopharyngeal carcinoma. BMC Cancer 2014;14:583.

-60 Desch A, Gebhardt C, Utikal J, Schneider SW: D-dimers in malignant melanoma: Association with prognosis and dynamic variation in disease progress. Int J Cancer 2017;140:914-921.

61 Watanabe A, Araki K, Hirai K, Kubo N, Igarashi T, Tsukagoshi M, Ishii N, Hoshino K, Kuwano H, Shirabe K: A Novel Clinical Factor, D-Dimer Platelet Multiplication, May Predict Postoperative Recurrence and Prognosis for Patients with Cholangiocarcinoma. Ann Surg Oncol 2016;23:886-891.

62 Liu Z, Guo H, Gao F, Shan Q, Li J, Xie H, Zhou L, Xu X, Zheng S: Fibrinogen and D-dimer levels elevate in advanced hepatocellular carcinoma: High pretreatment fibrinogen levels predict poor outcomes. Hepatol Res 2016;10.1111/hepr.12848

63 Wu J, Fu Z, Liu G, Xu P, Xu J, Jia X: Clinical significance of plasma D-dimer in ovarian cancer: A meta-analysis. Medicine (Baltimore) 2017;96:e7062.

64 Perisanidis C, Psyrri A, Cohen EE, Engelmann J, Heinze G, Perisanidis B, Stift A, Filipits M, Kornek G, Nkenke E: Prognostic role of pretreatment plasma fibrinogen in patients with solid tumors: A systematic review and meta-analysis. Cancer Treat Rev 2015;41:960-970.

-65 Steinbrecher KA, Horowitz NA, Blevins EA, Barney KA, Shaw MA, Harmel-Laws E, Finkelman FD, Flick MJ, Pinkerton MD, Talmage KE, Kombrinck KW, Witte DP, Palumbo JS: Colitis-associated cancer is dependent on the interplay between the hemostatic and inflammatory systems and supported by integrin alpha(M) beta(2) engagement of fibrinogen. Cancer Res 2010;70:2634-2643. 


\section{Cellular Physiology Cell Physiol Biochem 2018;45:1663-1676

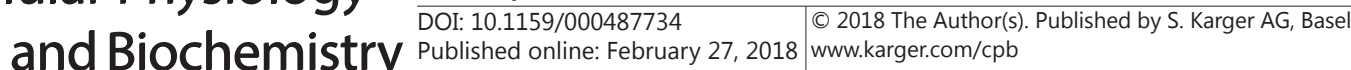 \\ Li et al.: Prognositc Role of D-Dimer in Solid Tumors: a Meta-Analysis}

66 Shu YJ, Weng H, Bao RF, Wu XS, Ding Q Cao Y, Wang XA, Zhang F, Xiang SS, Li HF, Li ML, Mu JS, Wu WG, Liu YB: Clinical and prognostic significance of preoperative plasma hyperfibrinogenemia in gallbladder cancer patients following surgical resection: a retrospective and in vitro study. BMC Cancer 2014;14:566.

67 Staton CA, Brown NJ, Lewis CE: The role of fibrinogen and related fragments in tumour angiogenesis and metastasis. Expert Opin Biol Ther 2003;3:1105-1120.

68 Alcalay A, Wun T, Khatri V, Chew HK, Harvey D, Zhou H, White RH: Venous thromboembolism in patients with colorectal cancer: incidence and effect on survival. J Clin Oncol 2006;24:1112-1118.

-69 Chew HK, Wun T, Harvey DJ, Zhou H, White RH: Incidence of venous thromboembolism and the impact on survival in breast cancer patients. J Clin Oncol 2007;25:70-76.

-70 Arora M, Wun T: Adverse impact of venous thromboembolism on patients with cancer. Semin Thromb Hemost 2014;40:313-318.

71 Ferroni P, Martini F, Portarena I, Massimiani G, Riondino S, La Farina F, Mariotti S, Guadagni F, Roselli M: Novel high-sensitive D-dimer determination predicts chemotherapy-associated venous thromboembolism in intermediate risk lung cancer patients. Clin Lung Cancer 2012;13:482-487.

72 Stender MT, Frokjaer JB, Larsen TB, Lundbye-Christensen S, Thorlacius-Ussing O: Preoperative plasma D-dimer is a predictor of postoperative deep venous thrombosis in colorectal cancer patients: a clinical, prospective cohort study with one-year follow-up. Dis Colon Rectum 2009;52:446-451.

73 Arpaia G, Carpenedo M, Verga M, Mastrogiacomo O, Fagnani D, Lanfredini M, Milani M, Cimminiello C: D-dimer before chemotherapy might predict venous thromboembolism. Blood Coagul Fibrinolysis 2009;20:170-175.

74 Shoji M, Hancock WW, Abe K, Micko C, Casper KA, Baine RM, Wilcox JN, Danave I, Dillehay DL, Matthews E, Contrino J, Morrissey JH, Gordon S, Edgington TS, Kudryk B, Kreutzer DL, Rickles FR: Activation of coagulation and angiogenesis in cancer: immunohistochemical localization in situ of clotting proteins and vascular endothelial growth factor in human cancer. Am J Pathol 1998;152:399-411.

-75 Nagy JA, Morgan ES, Herzberg KT, Manseau EJ, Dvorak AM, Dvorak HF: Pathogenesis of ascites tumor growth: angiogenesis, vascular remodeling, and stroma formation in the peritoneal lining. Cancer Res 1995;55:376-385.

-76 Blackwell K, Haroon Z, Broadwater G, Berry D, Harris L, Iglehart JD, Dewhirst M, Greenberg C: Plasma D-dimer levels in operable breast cancer patients correlate with clinical stage and axillary lymph node status. J Clin Oncol 2000;18:600-608.

-77 Zacharski LR, Henderson WG, Rickles FR, Forman WB, Cornell CJ, Jr., Forcier RJ, Edwards RL, Headley E, Kim SH, O'Donnell JF, et al.: Effect of warfarin anticoagulation on survival in carcinoma of the lung, colon, head and neck, and prostate. Final report of VA Cooperative Study \#75. Cancer 1984;53:2046-2052.

78 Klerk CP, Smorenburg SM, Otten HM, Lensing AW, Prins MH, Piovella F, Prandoni P, Bos MM, Richel DJ, van Tienhoven G, Buller HR: The effect of low molecular weight heparin on survival in patients with advanced malignancy. J Clin Oncol 2005;23:2130-2135.

79 Altinbas M, Coskun HS, Er O, Ozkan M, Eser B, Unal A, Cetin M, Soyuer S: A randomized clinical trial of combination chemotherapy with and without low-molecular-weight heparin in small cell lung cancer. J Thromb Haemost 2004;2:1266-1271. 